\title{
High accuracy determination of photoelectric crosssections, X-ray Absorption Fine Structure and nanostructure analysis of zinc selenide using the X-ray Extended Range Technique
}

\section{Daniel Sier ${ }^{1}$, Geoffrey P Cousland ${ }^{1}$, Ryan M Trevorah ${ }^{1}$, Ruwini S K Ekanayake ${ }^{1}$, Chanh Q Tran², James R Hester ${ }^{3}$, Christopher T Chantler ${ }^{1}$}

\author{
${ }^{1}$ University of Melbourne, Melbourne, Australia; \\ ${ }^{2}$ La Trobe University; \\ ${ }^{3}$ Australian Nuclear Science and Technology Organisation; \\ dsier@student.unimelb.edu.au
}

Measurements of mass attenuation coefficients and X-ray absorption fine structure (XAFS) of zinc selenide (ZnSe) are reported to accuracies typically better than $0.13 \%$. The high accuracy of the results presented here is due to our successful implementation of the X-ray Extended Range Technique (XERT), a relatively new methodology, which can be set up on most synchrotron X-ray beamlines. 561 attenuation coefficients were recorded in the energy range of $6.8 \mathrm{keV}$ to $15 \mathrm{keV}$ that was independently calibrated using powder diffractometry, with measurements concentrated at the zinc and selenium pre-edge, near edge and fine structure absorption edge regions. The removal of systematic effects as well as coherent (Thermal Diffuse) and incoherent (Compton) scattering processes produced very high accuracy values of photoelectrc attenuation which in turn yielded a detailed nanostructural analysis of room temperature ZnSe with full uncertainty propagation. Bond lengths, accurate to $0.003 \AA$ to $0.009 \AA$, or $0.1 \%$ to $0.3 \%$, are plausible and physical. Small variation from a crystalline structure suggests local dynamic motion beyond that of a standard crystal lattice, noting that XAFS is sensitive to dynamic correlated motion. The results obtained in this work are the most accurate to date with comparisons to theoretically determined values of the attenuation showing discrepancies from literature theory of up to $4 \%$, motivating further investigation into the origin of such discrepancies.

Keywords: XAFS, Diffuse Scattering, Zinc Selenide, Crystal Structure Determination, Powder Diffraction 